\title{
Prognostic role of nutritional status in elderly patients hospitalized for COVID-19: a monocentric study
}

\author{
Guerino Recinella ${ }^{1}$ (1) - Giovanni Marasco ${ }^{2}$. Giovanni Serafini ${ }^{1} \cdot$ Lorenzo Maestri $^{1}$ - Giampaolo Bianchi ${ }^{1,2}$. \\ Paola Forti ${ }^{1,2} \cdot$ Marco Zoli ${ }^{1,2}$
}

Received: 26 July 2020 / Accepted: 26 September 2020 / Published online: 8 October 2020

(c) The Author(s) 2020

\begin{abstract}
Background Symptomatic severe acute respiratory syndrome-coronavirus-2 (SARS-CoV-2) infection incidence is higher in the elderly patients. Pre-existing geriatric conditions such as comorbidity and frailty seem related to worse hospital outcomes. Aims To assess the role of nutritional status as an independent prognostic factor for in-hospital death in elderly patients. Methods Consecutive elderly patients (age $>65$ years) hospitalized for novel coronavirus disease (COVID-19) were enrolled. Demographics, laboratory and comorbidity data were collected. Nutritional status was evaluated using the Geriatric Nutritional Risk Index (GNRI). Uni- and multivariate Cox regression analyses to evaluate predictors for in-hospital death were performed.

Results One hundred and nine hospitalized elderly patients (54 male) were consecutively enrolled. At univariate analysis, age (HR 1.045 [CI 1.008-1.082]), cognitive impairment (HR 1.949 [CI 1.045-3.364]), C-reactive protein (HR 1.004 [CI 1.011-1.078]), lactate dehydrogenases (HR 1.003 [CI 1.001-1.004]) and GNRI moderate-severe risk category (HR 8.571 [CI 1.096-67.031]) were risk factors for in-hospital death, while albumin (HR 0.809 [CI 0.822-0.964]), $\mathrm{PaO}_{2} / \mathrm{FiO}_{2}$ ratio (HR 0.996 [CI 0.993-0.999]) and body mass index (HR 0.875 [CI 0.782-0.979]) were protective factors. Kaplan-Meier survival curves showed a significative higher survival in patients without GNRI moderate or severe risk category $(p=0.0013)$.

At multivariate analysis, $\mathrm{PaO}_{2} / \mathrm{FiO}_{2}$ ratio (HR 0.993 [CI 0.987-0.999], $p=0.046$ ) and GNRI moderate-severe risk category (HR 9.285 [1.183-72.879], $p=0.034$ ) were independently associated with in-hospital death.

Conclusion Nutritional status assessed by GNRI is a significative predictor of survival in elderly patients hospitalized for COVID-19. The association between GNRI and $\mathrm{PaO}_{2} / \mathrm{FiO}_{2}$ ratio is a good prognostic model these patients.
\end{abstract}

Keywords COVID-19 $\cdot$ Elderly $\cdot$ Nutrition $\cdot$ Geriatric nutritional risk index

\section{Introduction}

Guerino Recinella

guerino.recinella@gmail.com

$\triangle$ Giovanni Marasco

giovannimarasco89@gmail.com

1 Unit of Internal Medicine, Department of Medical and Surgical Sciences, S. Orsola- Malpighi University Hospital, University of Bologna, Via Pietro Albertoni 15, 40138 Bologna, Italy

2 Department of Medical and Surgical Sciences, S. Orsola-Malpighi Hospital, University of Bologna, Via Pietro Albertoni 15, 40138 Bologna, Italy
Since the beginning of the novel coronavirus disease (COVID-19) emergency in Italy, more than 200,000 infections and more than 30,000 deaths have been documented.

Although most patients suffer from a mild illness, a relatively high percentage of patients need to be hospitalized so the pandemic has put hospital systems under strain $[1,2]$. The majority of hospitalized patients are elderly [3].

These patients undergo to higher mortality mainly due to their frailty, the presence of comorbidity and high degree of disability [4-6]. However, only a few studies evaluated the epidemiological characteristics and the predictive factors of unfavourable outcomes in elderly patients with COVID-19 $[7,8]$. 
The alterations of the immune system of the elderly patients may have an important prognostic role. Indeed, the remodelling of the immune response observed among the elderly could explain the increased prevalence of more aggressive clinical manifestations of COVID-19 in these patients [9]. In particular, the state of chronic inflammation when not under control loses its defensive role and turns into a damaging state to the whole organism; the practical consequence is that inflamm-aging predicts frailty, and is associated with higher mortality.

Malnutrition is another possible explanation for the worse outcomes of elderly patients symptomatic COVID-19. This geriatric syndrome has a multifactorial aetiology and is strongly related to frailty and negative hospital outcomes in patients admitted with acute illnesses [10].

Although a prognostic role of nutritional status in elderly patients with COVID-19 has been hypothesized [11-13], to our knowledge, no study has assessed the prognostic value of nutritional status in elderly patients hospitalized for COVID-19.

We aimed to assess the prognostic role of nutritional status for in-hospital death of elderly patients hospitalized for COVID-19.

\section{Methods}

The study included 109 patients consecutively admitted to two COVID-19 units of Sant'Orsola-Malpighi University Hospital in Bologna between 30 March 30th and May 15th 2020. Inclusion criteria were (1) age $\geq 65$; (2) diagnosis of COVID-19 based on the detection of severe acute respiratory syndrome-coronavirus-2 (SARS-CoV-2) on reverse transcriptase polymerase chain reaction (RT-PCR) from the nasopharyngeal swab. Exclusion criteria were the presence of terminal neoplasia and the exclusively clinical and radiological diagnosis of COVID-19 without laboratory confirmation. Demographics, past medical history, and clinical and laboratory data on admission were recorded by patient's medical record. Comorbidity was assessed using the Charlson Comorbidity Index (CCI) [14]. Clinical and laboratory indicators of severity for SARS-COV-2 infection included lymphocytes, lactate dehydrogenases (LDH), C-reactive protein, D-dimer, and partial pressure of oxygen/fraction of inspired oxygen ratio $\left(\mathrm{PaO}_{2} / \mathrm{FiO}_{2}\right)$.

Nutritional status was evaluated using the Geriatric Nutritional Risk Index (GNRI) within $48 \mathrm{~h}$ of admission. The GNRI is a simple nutritional screening tool used to evaluate nutrition-related risk in surgical and medical patients $[15,16]$. The index was calculated as follows: $\mathrm{GNRI}=1.489 \times$ serum albumin $(\mathrm{g} / \mathrm{L})+41.7 \times$ present weight/ideal weight $(\mathrm{kg})$. Ideal body weight was derived using the equations of Lorentz [17]: ideal weight for men $=0.75 \times$ height $(\mathrm{cm})-62.5$, ideal weight for women $=0.60 \times$ height $(\mathrm{cm})-40$. According with previous study [18], three categories were identified: no risk (GNRI > 98), low risk (GRNI 92-98) and severe-moderate risk $($ GNRI $<92)$.

Patients' follow-up started at admission and was carried out until hospital discharge or death. The evaluated outcome was in-hospital death. The study was conducted according to the declaration of Helsinki's ethical principles for medical research involving human subjects. Informed consent was obtained from each patient (or from patient's relatives if the subject was disabled). The protocol was reviewed and approved by the Ethics Committee of the S. Orsola University Hospital (Protocol number 512/2020/Oss/AOUBO).

\section{Statistical analysis}

Data were reported as median with interquartile range (IQR) for continuous variables and numbers and percentages for categorical variables. Comparisons between patients grouped by vital status at discharge were analyzed by Fischer, Chi-square or Mann-Whitney tests when appropriate. Subsequently, the same variables were tested as independent variables associated with in-hospital death. First, several univariate Cox regression analyses were performed considering statistically significant those variables with $p$ value less than 0.1 . Subsequently, only the variables significantly associated with the in-hospital death in univariate analyses were entered into a multivariate model, excluding collinear variables. Finally, the best multivariate model was identified, adopting a backward elimination procedure. The estimated hazard ratios (HR) with their $95 \%$ confidence intervals $(95 \%$ CI) were calculated; $p$ values less than 0.05 were considered statistically significant. The results obtained from multivariate analysis, in the presence of two or more co-variates influencing the risk, were translated in graphic form through the use of nomograms for Cox regression analyses.

A Kaplan-Meier survival curve to estimate the survival according to GNRI categories (no risk/low risk vs moderate risk/severe risk) was constructed; the statistical significance of differences between GNRI categories was tested with the log-rank test. Statistical analyses were performed using Stata/SE (Version 13.0; Stata Corp, Texas, United States of America) for Windows.

\section{Results}

One-hundred and nine patients (109, 54 male 51.4\%) were consecutively included in our study. The median age was 83 years (76-91.5). During a median follow-up of 11 (8-15) days, 43 (39.4\%) patients died. Most of the patients enrolled reported other underlying comorbidities: arterial 
hypertension (75.2\%), cognitive impairment (44\%) and atrial fibrillation (29.4\%) were the most common. During hospitalization, six patients $(5.5 \%)$ were transferred to the intensive care unit (ICU). None $(0 \%)$ of patients transferred to ICU had moderate to severe GNRI, while 67 (65\%) patients in the group "no transfer to ICU” had moderate to severe GNRI.

\section{Demographics and clinical status}

Table 1 shows characteristics of patients grouped by vital status at discharge. In-hospital death was associated with higher age, longer hospital stay, cognitive impairment, and clinical and laboratory indicators of more severe disease (higher prevalence of dyspnea on admission, higher C-reactive protein and $\mathrm{LDH}$, lower $\mathrm{PaO}_{2} / \mathrm{FiO}_{2}$ ).

\section{Assessment of nutritional status}

Considering the nutritional parameters, lower values of body weight $(p=0.001)$, BMI $(p=0.002)$ and albumin $(p<0.001)$ were found in patients experiencing in-hospital death. On the other hand, higher values of GNRI were found in surviving patients $(p<0.001)$ and a higher prevalence of GNRI moderate-severe risk category was found in the in-hospital death group $(p=0.004)$. Nutritional parameters are summarized in Table 1.
Table 1 Differences in demographic, clinical and laboratory findings between group of patients experiencing in-hospital death and not

\begin{tabular}{|c|c|c|c|}
\hline & $\begin{array}{l}\text { No in-hospital death }(n=66), \\
n(\%) \text { or median }[\mathrm{IQR}]\end{array}$ & $\begin{array}{l}\text { In-hospital death }(n=43), \\
n(\%) \text { or median }[\mathrm{IQR}]\end{array}$ & $p$ value \\
\hline Age & 79 [74-92] & 85.5 [79-86.7] & 0.007 \\
\hline Male & $32(48.5)$ & $22(51.2)$ & 0.785 \\
\hline Length of hospital stay, day & $8[7-15]$ & $11[8-15]$ & 0.010 \\
\hline \multicolumn{4}{|l|}{ Comorbidity } \\
\hline Charlson Comorbidity Index & $4[3-6]$ & $4.5[2.2-8.7]$ & 0.817 \\
\hline Cognitive impairment & $21(31.8)$ & $27(62.8)$ & 0.001 \\
\hline Previous stroke & $7(10.6)$ & $10(23.3)$ & 0.075 \\
\hline Arterial hypertension & $49(74.2)$ & $43(76.7)$ & 0.767 \\
\hline Diabetes & $16(24.2)$ & $8(18.6)$ & 0.488 \\
\hline Atrial fibrillation & $19(28.8)$ & $13(30.2)$ & 0.871 \\
\hline Coronary heart disease & $9(13.6)$ & $6(14)$ & 0.963 \\
\hline Chronic heart failure & $9(13.6)$ & $8(18.6)$ & 0.485 \\
\hline COPD & $12(18.2)$ & $12(27.9)$ & 0.231 \\
\hline CKD & $15(22.7)$ & $8(18.6)$ & 0.606 \\
\hline \multicolumn{4}{|l|}{ Symptoms } \\
\hline Fever & $43(65.2)$ & $25(58.1)$ & 0.460 \\
\hline Dyspnea & $20(30.3)$ & $30(69.8)$ & $<0.001$ \\
\hline Cough & $24(36.4)$ & $14(32.6)$ & 0.684 \\
\hline Asthenia & $21(31.8)$ & $14(32.5)$ & 0.653 \\
\hline \multicolumn{4}{|c|}{ Laboratory features and nutritional parameters } \\
\hline $\mathrm{PaO}_{2} / \mathrm{FiO}_{2}$ & $319[276-347]$ & $254[175-286]$ & 0.006 \\
\hline Lymphocytes, $10^{9} / \mathrm{L}$ & $1.01[0.81-1.32]$ & $0.72[0.45-1.12]$ & 0.071 \\
\hline C-reactive protein, $\mathrm{mg} / \mathrm{dL}$ & $6.35[1.09-11.32]$ & $13.38[4.24-19.14]$ & 0.003 \\
\hline $\mathrm{LDH}, \mathrm{U} / \mathrm{L}$ & $222[188-310]$ & $266[197-381]$ & 0.012 \\
\hline D-dimer, $\mu \mathrm{g} / \mathrm{mL}$ & $1.12[0.5-2.49]$ & $1.99[0.79-3.79]$ & 0.140 \\
\hline $\mathrm{GRF}, \mathrm{mL} / \mathrm{min}$ & $67[33-81]$ & $46.5[39.7-78.2]$ & 0.281 \\
\hline Albumin, g/L & $31.6[28.3 .8-35.3]$ & $26.3[23.9-30.7]$ & $<0.001$ \\
\hline Weight, kg & $73[63-80]$ & $55[45-73.5]$ & 0.001 \\
\hline BMI, $\mathrm{kg} / \mathrm{m}^{2}$ & $25.7[22.5-28.5]$ & $20.3[16-23.9]$ & 0.002 \\
\hline GNRI & $95[88-103]$ & $82[69-87.5]$ & $<0.001$ \\
\hline No risk & $26(39.4)$ & $4(9.3)$ & 0.058 \\
\hline Low risk & $12(18.2)$ & $0(0)$ & 0.124 \\
\hline Moderate-severe risk & $28(42.4)$ & $39(90.7)$ & 0.004 \\
\hline
\end{tabular}

$n=$ numbers, $I Q R$ interquartile range, $B M I$ body mass index, $C O P D$ chronic obstructive pulmonary disease, $C K D$ chronic kidney disease, $\mathrm{FiO}_{2}$ fraction of inspired oxygen, GNRI Geriatric Nutrition Risk Index, GRF glomerular filtration rate, $\mathrm{LDH}$ lactate dehydrogenases, $\mathrm{PaO}_{2}$ partial pressure of oxygen 


\section{Independent in-hospital death predictors}

Among all variables evaluated in the univariate Cox regression analysis (Table 2), age, cognitive impairment, $\mathrm{C}$-reactive protein, $\mathrm{LDH}$, and GNRI moderate-severe risk category (HR 8.571 [CI 1.096-67.031] $p=0.041$ ) were associated with in-hospital death, while albumin, body mass index, and $\mathrm{PaO}_{2} / \mathrm{FiO}_{2}$ ratio showed a protective role.

Kaplan-Meier survival curves according to the GNRI were estimated (Fig. 1). A higher statistically significant survival was found in the group without risk or low risk according to GNRI, compared to those with moderate risk or severe risk $(p=0.003)$.

Multivariate Cox regression analysis showed that GNRI moderate-severe risk category (HR 9.285 [1.183-72.879], $p=0.034$ ) and $\mathrm{PaO}_{2} / \mathrm{FiO}_{2}$ ratio (HR 0.993 [CI $0.987-0.999$ ], $p=0.046$ ) were the only independent predictors of in-hospital death. The results of the multivariate Cox regression analysis were graphically reported in a nomogram showing the different probability of survival at 7, 14, and 21 days after hospital admission (Fig. 2).

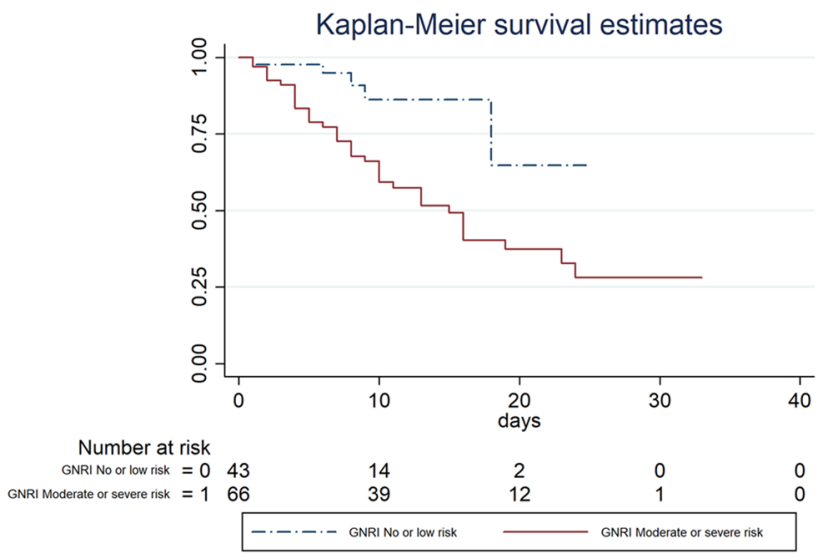

Fig. 1 Kaplan-Meier survival curves according to the GNRI groups: no or low risk group versus moderate or high risk group

\section{Discussion}

The main result of our study is that impaired nutritional status, assessed by the GNRI, together with the $\mathrm{PaO}_{2} / \mathrm{FiO}_{2}$ ratio, is an independent predictor of in-hospital mortality in elderly patients with symptomatic SARS-CoV-2 infection.
Table 2 Univariate and multivariate analyses for independent variables associated with in-hospital death

\begin{tabular}{|c|c|c|c|c|}
\hline & \multicolumn{2}{|l|}{ Univariate } & \multicolumn{2}{|l|}{ Multivariate } \\
\hline & $\operatorname{HR}(95 \% \mathrm{CI})$ & $p$ value & $\mathrm{HR}(95 \% \mathrm{CI})$ & $p$ value \\
\hline Age & $1.045(1.008-1.082)$ & 0.014 & & \\
\hline Sex & $1.109(0.609-2.018)$ & 0.734 & & \\
\hline Charlson Comorbidity Index & $0.963(0.835-1.111)$ & 0.612 & & \\
\hline Cognitive impairment & $1.949(1.045-3.364)$ & 0.036 & & \\
\hline Coronary heart disease & $1.018(0.428-2.418)$ & 0.967 & & \\
\hline Atrial fibrillation & $1.111(0.579-2.132)$ & 0.750 & & \\
\hline Arterial hypertension & $1.006(0.495-2.047)$ & 0.985 & & \\
\hline Diabetes & $0.751(0.347-1.622)$ & 0.466 & & \\
\hline Previous stroke & $1.431(0.704-2.908)$ & 0.322 & & \\
\hline Chronic heart failure & $1.024(0.472-2.223)$ & 0.951 & & \\
\hline CKD & $1.251(0.572-2.732)$ & 0.574 & & \\
\hline COPD & $1.434(0.735-2.799)$ & 0.291 & & \\
\hline Lymphocytes & $1.138(0.834-1.554)$ & 0.415 & & \\
\hline C-reactive protein & $1.044(1.011-1.078)$ & 0.008 & & \\
\hline LDH & $1.003(1.001-1.004)$ & $<0.001$ & & \\
\hline D-dimer & $1.019(0.976-1.065)$ & 0.383 & & \\
\hline GRF & $0.994(0.983-1.004)$ & 0.286 & & \\
\hline Albumin & $0.890(0.822-0.964)$ & 0.004 & & \\
\hline $\mathrm{PaO}_{2} / \mathrm{FiO}_{2}$ & $0.996(0.993-0.999)$ & 0.008 & $0.993(0.987-0.999)$ & 0.046 \\
\hline BMI & $0.875(0.782-0.979)$ & 0.020 & & \\
\hline GNRI (moderate-severe risk) & $8.571(1.096-67.031)$ & 0.041 & $9.285(1.183-72.879)$ & 0.034 \\
\hline
\end{tabular}

$B M I$ body mass index, $\mathrm{CKD}$ chronic kidney disease, $\mathrm{COPD}$ chronic obstructive pulmonary disease, $\mathrm{FiO}_{2}$ fraction of inspired oxygen, GFR glomerular filtration rate, GNRI Geriatric Nutrition Risk Index, $L D H$ lactate dehydrogenases, $\mathrm{PaO}_{2}$ partial pressure of oxygen 
Fig. 2 A nomogram with GNRI values and $\mathrm{PaO} 2 / \mathrm{FiO} 2$ ratio showing the different probability of survival at 7,14 , and 21 days after hospital admission

\section{Probability of Survival}

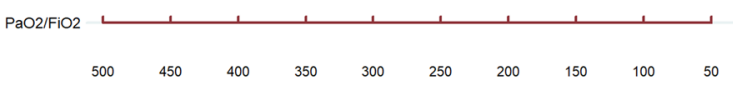

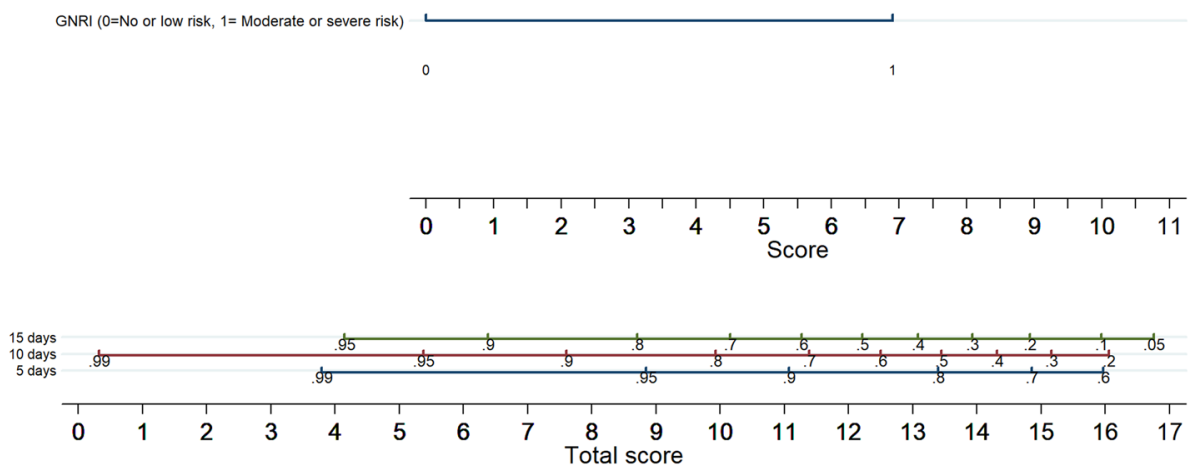

To our knowledge, this is the first study reporting data on the predictive ability of nutritional scales in elderly patients hospitalized for SARS-CoV-2 infection.

We assessed nutritional status using GNRI which is a score already validated in the literature both in surgical and clinical settings $[15,16,19]$. Our study shows that GNRI is also a possible prognostic tool for mortality of elderly patients with COVID-19. In fact, an increased risk of in-hospital mortality was present in the GNRI moderate-severe risk group with a ninefold increase in risk in the multivariate model (HR 9.285 [1.183-72.879], $p=0.034$ ). Thus, the pre-hospital status of the elderly patient, not only in terms of comorbidity, but also in terms of nutritional parameters, represents one of the main prognostic factors in patients hospitalized for COVID-19.

In line with these results, our elderly population showed higher albuminemia values in the group of patients not experiencing in-hospital death $(p<0.001)$; this protective relationship was also confirmed by the univariate analysis (HR 0.809 [CI 0.822-0.964]). In addition, a peculiar result of our study concerns BMI; previous studies in not age-selected population reported that an higher BMI was associated with unfavourable outcomes in subjects with COVID-19, in particular higher risk of hospitalization, risk of ICU transfer and length of ICU stay, and finally of death [20]. Surprisingly, in our study, a higher values of BMI showed a protective role; this may probably be due to the study design, including only subjects with age over 65 years. Indeed, in the elderly population, a higher BMI value could be translated in a better nutritional status [21], thus explaining our findings.

Our observations agree with previous evidence that malnutrition is associated with an increased mortality from both infections and chronic diseases [21, 22]. There are several reasons for this association, including the close association between malnutrition and immune system deficit [10, 23].

We, therefore, believe that maximum attention should be paid to COVID-19 elderly patient with malnutrition and where possible start quickly a supplementary feeding.

Another factor independently associated with intra-hospital mortality was the $\mathrm{PaO}_{2} / \mathrm{FiO}_{2}$ ratio. This finding agrees with previous studies in adult populations [24,25] and may be due to a greater pulmonary involvement in patients with low values of this ratio.

Another novelty of this study is represented by the use of nomograms that were elaborated for evaluating the probability of survival of elderly patients hospitalized for COVID19; the nomogram provided represent an easy-handling tool for rapidly calculating the probability of survival for a single patient in the era of tailored medicine.

Only a few previous researches described the clinical and epidemiological characteristics of elderly patients hospitalized for SARS-CoV-2 infection [5, 6, 8, 26]. Similar to our study, most patients were characterized by a high number of chronic comorbid condition and pre-existent functional disability.

In our study, we did not assess cognitive impairment on standardized scales, but data were collected according to the medical history. Nevertheless, our results show that cognitive impairment was highly prevalent among patients who died before discharge; however, multivariable analyses did not confirm cognitive impairment as an independent predictor. In other studies assessing the predictive value of cognitive impairment in COVID-19 patients, it has been reported to be a predictive factor for unfavourable outcomes [26, 27]. Several reasons may explain this association and why it disappeared in favor of GNRI and $\mathrm{PaO}_{2} / \mathrm{FiO}_{2}$ ratio. First, patients with cognitive impairment have poor compliance 
with prescriptions [28] such as oxygen therapy; in addition, cognitive impairment is closely related to sarcopenia and malnutrition, which in turn is associated with increased adverse outcomes [29].

In our elderly population, the most frequent COVID-19 clinical symptoms were fever, cough and dyspnea. Notably, dyspnea prevalence was significantly higher in patients experiencing in-hospital death. This suggests that the greater the respiratory involvement, the worse the outcome of COVID19 patients.

Our study has some limitations. First, due to the monocentric design, the enrolled population may be not representative of the elderly patients hospitalized for COVID-19. A second limitation is the lack of data about other relevant parameters of body fat and muscle mass (e.g. waist circumference, muscle thickness). However, given the dramatic nature of the situation, it was difficult to collect complex nutritional parameters even to minimise the risk for health workers.

In addition, our multivariate analysis may be affected by an overfitting variable bias since we reported only 43 death; moreover, our predictive model still needs an internal and external validation.

However, our study has also several strengths. This is the first report including nutritional parameters as a prognostic factors in elderly patients hospitalized for COVID-19; in addition, the assessment of nutritional status has been carried out using a simple, rapid and effective score especially in high infectious risk contexts where complex measurements cannot be carried out. Finally, we provided a nomogram able to personalize the individual probability of survival of each geriatric patient hospitalized for COVID-19. Future larger and multicentric studies are needed to validate our results on the prognostic value of GNRI in predicting in-hospital death and to further explore the role of other nutritional parameters in elderly patients hospitalized for COVID-19.

\section{Conclusion}

Nutritional status assessed by GNRI is an independent prognostic factor for in-hospital mortality. The association of GNRI with a respiratory parameter $(\mathrm{PaO} 2 / \mathrm{FiO} 2)$ provides a comprehensive prognostic tool for predicting adverse outcomes in elderly COVID-19 patients.

Author contributions Study concept: RG, MG, ML, and SG. Drafting of the manuscript: RG, MG, and FP. Revision of the manuscript: FP and ZM. Approval of the manuscript: all authors. Data analysis: MG and RG.
Funding Open access funding provided by Alma Mater Studiorum Università di Bologna within the CRUI-CARE Agreement.

\section{Compliance with ethical standards}

Conflict of interest On behalf of all authors, the corresponding author states that there is no conflict of interest.

Statement of human and animal rights All procedures and the research protocol have been approved by the locally appointed ethics committee of the University of Bologna. The experimental protocol was designed in accordance with the Declaration of Helsinki (1964).

Informed consent Informed consent was obtained from all individual participants included in the study.

Open Access This article is licensed under a Creative Commons Attribution 4.0 International License, which permits use, sharing, adaptation, distribution and reproduction in any medium or format, as long as you give appropriate credit to the original author(s) and the source, provide a link to the Creative Commons licence, and indicate if changes were made. The images or other third party material in this article are included in the article's Creative Commons licence, unless indicated otherwise in a credit line to the material. If material is not included in the article's Creative Commons licence and your intended use is not permitted by statutory regulation or exceeds the permitted use, you will need to obtain permission directly from the copyright holder. To view a copy of this licence, visit http://creativecommons.org/licenses/by/4.0/.

\section{References}

1. Smith MJ, Hayward SA, Innes SM et al (2020) Point-of-care lung ultrasound in patients with COVID-19-a narrative review. Anaesthesia. https://doi.org/10.1111/anae.15082

2. Wu C, Chen X, Cai Y et al (2020) Risk factors associated with acute respiratory distress syndrome and death in patients with coronavirus disease 2019 pneumonia in Wuhan. JAMA Intern Med, China. https://doi.org/10.1001/jamainternmed.2020.0994

3. Xu XW, Wu XX, Jiang XG et al (2020) Clinical findings in a group of patients infected with the 2019 novel coronavirus (SARS-Cov-2) outside of Wuhan, China: retrospective case series. BMJ. https://doi.org/10.1136/bmj.m606

4. Onder G, Rezza G, Brusaferro S (2020) Case-fatality rate and characteristics of patients dying in relation to COVID-19 in Italy. JAMA. https://doi.org/10.1001/jama.2020.4683

5. Li P, Chen L, Liu Z et al (2020) Clinical features and short-term outcomes of elderly patients with COVID-19. Int J Infect Dis. https://doi.org/10.1016/j.ijid.2020.05.107

6. Liu K, Chen Y, Lin R et al (2020) Clinical features of COVID-19 in elderly patients: a comparison with young and middle-aged patients. J Infect. https://doi.org/10.1016/j.jinf.2020.03.005

7. Guo Y, Liu X, Deng M et al (2020) Epidemiology of COVID19 in older persons, Wuhan, China. Age Ageing. https://doi. org/10.1093/ageing/afaa145

8. Lee JY, Kim HA, Huh K et al (2020) Risk factors for mortality and respiratory support in elderly patients hospitalized with COVID-19 in Korea. J Korean Med Sci. https://doi.org/10.3346/ jkms.2020.35.e223

9. Cunha LL, Perazzio SF, Azzi J et al (2020) Remodeling of the immune response with aging: immunosenescence and its potential impact on COVID-19 immune response. Front Immunol. https:// doi.org/10.3389/fimmu.2020.01748 
10. Volkert D, Beck AM, Cederholm T et al (2019) Management of malnutrition in older patients-current approaches, evidence and open questions. J Clin Med. https://doi.org/10.3390/jcm8070974

11. Lidoriki I, Frountzas M, Schizas D (2020) Could nutritional and functional status serve as prognostic factors for COVID19 in the elderly? Med Hypotheses. https://doi.org/10.1016/j. mehy.2020.109946

12. Li T, Zhang Y, Gong C et al (2020) Prevalence of malnutrition and analysis of related factors in elderly patients with COVID-19 in Wuhan, China. Eur J Clin Nutr. https://doi.org/10.1038/s4143 0-020-0642-3

13. Recinella G, Marasco G, Di Battista A et al (2020) Prognostic role of nutritional status in elderly patients hospitalized for COVID19. Med Hypotheses 144:110016. https://doi.org/10.1016/j. mehy.2020.110016

14. Charlson ME, Pompei P, Ales KL et al (1987) A new method of classifying prognostic comorbidity in longitudinal studies: development and validation. J Chronic Dis. https://doi. org/10.1016/0021-9681(87)90171-8

15. Bouillanne O, Morineau G, Dupant C et al (2005) Geriatric Nutritional Risk Index: a new index for evaluating at-risk elderly medical patients. Am J Clin Nutr. https://doi.org/10.1093/ajcn/82.4.777

16. Nishi I, Seo Y, Hamada-Harimura Y et al (2019) Geriatric nutritional risk index predicts all-cause deaths in heart failure with preserved ejection fraction. ESC Heart Fail. https://doi.org/10.1002/ ehf2.12405

17. Cereda E, Pedrolli C (2009) The geriatric nutritional risk index. Curr Opin Clin Nutr Metab Care. https://doi.org/10.1097/ MCO.0b013e3283186f59

18. Zhao Y, Xia X, Xie D et al (2020) Geriatric Nutritional Risk Index can predict postoperative delirium and hospital length of stay in elderly patients undergoing non-cardiac surgery. Epidemiol Clin Pract Health. https://doi.org/10.1111/ggi.13963

19. Xu J, Zhou X, Zheng C (2019) The geriatric nutritional risk index independently predicts adverse outcomes in patients with pyogenic liver abscess. BMC Geriatr. https://doi.org/10.1186/s1287 7-019-1030-5

20. Leung YY, Allen JC, Noviani M et al (2015) Association between body mass index and risk of total knee replacement, the Singapore
Chinese Health Study. Osteoarthr Cartil. https://doi.org/10.1016/j. joca.2014.10.011

21. Tallarico V, Recinella G, Magalotti D et al (2020) Prognostic value of non-alcoholic fatty liver disease in the elderly patients. Aging Clin Exp Res. https://doi.org/10.1007/s40520-020-01487 $-2$

22. Lee JS, Choi HS, Ko YG et al (2013) Performance of the Geriatric Nutritional Risk Index in predicting 28-day hospital mortality in older adult patients with sepsis. Clin Nutr. https://doi. org/10.1016/j.clnu.2013.01.007

23. Norman K, Pichard C, Lochs H et al (2008) Prognostic impact of disease-related malnutrition. Clin Nutr. https://doi.org/10.1016/j. clnu.2007.10.007

24. Chen T, Wu D, Chen $\mathrm{H}$ et al (2020) Clinical characteristics of 113 deceased patients with coronavirus disease 2019: Retrospective study. BMJ. https://doi.org/10.1136/bmj.m1091

25. Yang X, Yu Y, Xu J et al (2020) Clinical course and outcomes of critically ill patients with SARS-CoV-2 pneumonia in Wuhan, China: a single-centered, retrospective, observational study. Lancet Respir Med. https://doi.org/10.1016/S2213-2600(20)30079-5

26. Niu S, Tian S, Lou J et al (2020) Clinical characteristics of older patients infected with COVID-19: a descriptive study. Arch Gerontol Geriatr. https://doi.org/10.1016/j.archger.2020.104058

27. Covino M, De Matteis G, Santoro M et al (2020) Clinical characteristics and prognostic factors in COVID-19 patients aged $\geq 80$ years. Geriatr Gerontol Int. https://doi.org/10.1111/ggi.13960

28. Insel K, Morrow D, Brewer B et al (2006) Executive function, working memory, and medication adherence among older adults. J Gerontol Ser B Psychol Sci Soc Sci. https://doi.org/10.1093/ geronb/61.2.P102

29. Cruz-Jentoft AJ, Sayer AA (2019) Sarcopenia. Lancet. https://doi. org/10.1016/S0140-6736(19)31138-9

Publisher's Note Springer Nature remains neutral with regard to jurisdictional claims in published maps and institutional affiliations. 